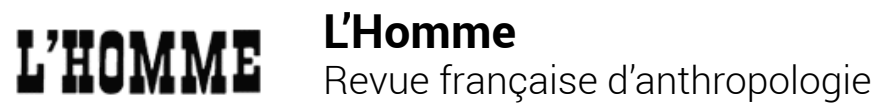

163 | juillet-sptembre 2002

De la légende au mythe. Parole, langue et pensée

\section{Alban Bensa \& Isabelle Leblic, s. dir., En pays kanak. Ethnologie, linguistique, archéologie, histoire de la Nouvelle-Calédonie}

Paris, Éditions de la Maison des sciences de l'homme, 2000, 368 p., ill., tabl., cartes (Mission du patrimoine ethnologique. « Ethnologie de la France ", Cahier 14)

\section{Christine Demmer}

\section{(2) OpenEdition}

\section{Édition électronique}

URL : http://journals.openedition.org/lhomme/12761

DOI : 10.4000//homme.12761

ISSN : 1953-8103

Éditeur

Éditions de l'EHESS

\section{Édition imprimée}

Date de publication : 21 juin 2002

Pagination : 307-309

ISBN : 2-7132-1771-7

ISSN : 0439-4216

Référence électronique

Christine Demmer, «Alban Bensa \& Isabelle Leblic, s. dir., En pays kanak. Ethnologie, linguistique, archéologie, histoire de la Nouvelle-Calédonie », L'Homme [En ligne], 163 | juillet-sptembre 2002, mis en ligne le 10 juillet 2007, consulté le 24 septembre 2020. URL : http://journals.openedition.org//homme/ 12761 ; DOI : https://doi.org/10.4000//homme.12761

Ce document a été généré automatiquement le 24 septembre 2020.

(C) École des hautes études en sciences sociales 


\section{Alban Bensa \& Isabelle Leblic, s. dir., En pays kanak. Ethnologie, linguistique, archéologie, histoire de la Nouvelle- Calédonie}

Paris, Éditions de la Maison des sciences de l'homme, 2000, 368 p., ill., tabl., cartes (Mission du patrimoine ethnologique. « Ethnologie de la France ", Cahier 14)

\section{Christine Demmer}

LES RECHERCHES anthropologiques sur les sociétés kanakes ont longtemps été rares, représentées par deux chercheurs, Maurice Leenhardt et Jean Guiart. Lorsque la revendication indépendantiste kanake s'exprima dans les années 1970, le manque d'études se fit sentir. Jean-Marie Tjibaou, soucieux de lier sa démarche politique à la compréhension de la culture kanake alors dénigrée - voire niée -, insista sur la nécessité d'une relance des recherches. C'est dans ce cadre qu'à partir des Accords de Matignon (1988) plusieurs programmes furent lancés, l'un par l'ORSTOM, l'autre par l'EHESS à travers le programme ESK (Études des sociétés kanakes) financé par les ministères des DOM-TOM et de la Culture (Mission du Patrimoine ethnologique). Ce livre est l'un des aboutissements de ce dernier programme. Il correspond à l'engagement de nouveaux chercheurs sur des sujets et des lieux d'enquêtes inédits : la ville, l'école, la santé, le politique contemporain, l'histoire, qui ont par ailleurs donné naissance à des livres (Isabelle Merle, Michel Naepels) et à des thèses (Elsa Faugère, Dorothée Dussy, Marie Pineau-Salaün, Charles Illouz, Marie Lepoutre, Yoram Mouchenik, Éric Soriano), ou à la poursuite de travaux engagés auparavant (Alban Bensa, Isabelle Leblic, Jean-Claude Rivierre, Christophe Sand). A posteriori, En pays kanak apparaît donc comme un état des lieux - plus ou moins exhaustif - de l'orientation des recherches menées en Nouvelle-Calédonie durant la période que couvrirent les Accords de Matignon (1988-1998), par des ethnologues, des linguistes, des historiens, des archéologues, un politologue et une sociologue. 
2 C'est autour d'une commune attention portée aux transformations sociales du monde kanak que ces textes se fédèrent. Loin de toute approche essentialiste, ils invitent à évaluer l'extraordinaire dynamisme des Kanaks qui ont su faire face à la colonisation en conjuguant leurs références sociopolitiques aux références exogènes (qu'elles soient imposées par la contrainte ou librement choisies), recréant ainsi, au sein des réserves où ils furent cantonnés, des rapports sociaux inédits. Cette capacité d'innovation, qui n'est pas propre à cette société mais qui dans ce contexte particulièrement contraignant mérite d'être souligné, reste toujours d'actualité à l'heure du nationalisme et des nécessaires mutations qu'il induit.

Dans cette perspective constructiviste, la première partie rassemble des textes qui témoignent de la tension (au principe même de toute possibilité de changement) entre références et pratiques sociales. Certains d'entre eux montrent comment des logiques sociales nouvelles se sont élaborées à partir d'une histoire particulière, d'autres de quelle manière, dans la pratique, certaines références sont utilisées et contournées. Alban Bensa explique comment le modèle politique de la chefferie territoriale centralisée s'est constitué dans la vallée de Koné avec l'appui des militaires, des missionnaires et de l'administration au cours des premières décennies de contact; Isabelle Leblic se penche sur le rôle de l'adoption et des transferts d'enfants dans l'élaboration de moitiés intermariantes à Ponérihouen; Françoise Ozanne-Rivierre étudie l'évolution des langues kanakes observée au regard de quelques termes de parenté; Dominique Bretteville analyse l'idéologie de l'organisation sociale de la chefferie paimboa; Marie-Hélène Teulières-Preston décrit les conflits qui existent entre droits maritimes kanaks et droit français ; enfin, Dorothée Dussy rend compte des types de légitimations invoquées par les Kanaks pour revendiquer le sol nouméen.

4 La deuxième partie propose un retour sur l'histoire passée, notamment sur la politique coloniale à travers certaines de ses institutions. Elle permet de mieux comprendre la façon dont les Kanaks se sont inscrits dans le dispositif colonial (voir sur ce point l'origine de la constitution des réserves par Isabelle Merle et le rôle des Missions lors de l'instauration du suffrage universel par Éric Soriano, ainsi quela politique d'insertion scolaire au temps de l'indigénat par Marie Pineau-Salaün). On voit comment, en dépit des contraintes et des bouleversements occasionnés (introduction de maladies et chute démographique évoquées par Christophe Sand, Jacques Bole et André Ouetcho), les Kanaks réagirent en réélaborant leurs rapports sociaux à lumière des situations présentes (comme le montre Charles Illouz à propos de la fabrication du chef à Maré et de l'adaptation de l'institution politique de la chefferie à certaines représentations chrétiennes).

5 La troisième et dernière partie rend compte de mutations plus contemporaines envisagées en milieu rural. Isabelle Brill évoque la tentative de fixation écrite d'une langue orale de l'extrême nord de la Grande Terre; Marie Lepoutre témoigne de l'appréhension syncrétique de la médecine aux îles Loyauté; Christine Salomon s'intéresse à la lutte des femmes contre la domination masculine dans les aires linguistiques ajië et paîci. D’autres textes renseignent sur un aspect méconnu de la réalité kanake, à savoir la nature des liens qu'entretiennent les Kanaks avec la ville et leurs pratiques en milieu urbain. Christine Hamelin révèle un ancrage et des expériences nouvelles en ville, tandis que Michel Naepels analyse diverses logiques migratoires à partir de l'exemple de la région de Houaïlou. 
6 La grande disparité thématique et géographique des études est l'un des atouts du livre dans la mesure où elle ouvre une large perspective de connaissances sur le monde kanak d'hier et d'aujourd'hui. Tous ces travaux sont le fruit d'expériences de terrain qui permettent de saisir des nuances, d'une région à l'autre, dans les modalités d'organisation des rapports sociaux et de saisir la spécificité des histoires coloniales locales. Toutefois, l'absence de fil conducteur commun à ces recherches - autre que méthodologique - empêche de dégager clairement, dans leur diversité et leur articulation, les grandes lignes des enjeux actuels de la pratique kanake issus de la confrontation d'univers sociaux différents. Un projet de recherche initié dans le contexte d'émancipation kanak aurait pu, à partir d'un tel cadre, atteindre mieux cet objectif en s'attachant explicitement à en décrire les racines, voire les ressorts, et à analyser la nature des transformations accélérées qu'il entraîne dans les différents domaines étudiés.

\section{AUTEUR}

CHRISTINE DEMMER

EHESS, Genèse et transformation des mondes sociaux, Paris. 